\title{
Communication Scheme to Construct Self-Organization IoT Network in Heterogeneous IoT Environments
}

\author{
Joosang Youn \\ Dept. of Industrial ICT Engineering, Dong-Eui University \\ 176, Eomgwangno, Busan jin-gu, Busan, 47340, South Korea \\ jsyoun@deu.ac.kr
}

\begin{abstract}
Recently, various IoT applications are developed in heterogeneous IoT environments which consist of the IoT devices with various network interfaces. This paper proposes novel communication scheme for self-organization IoT networking in homogeneous IoT and heterogeneous IoT environments. Particularly, this paper describes the concept of selforganization IoT networking in heterogeneous IoT environments. It also describes the architecture model for self-organization IoT networking. The proposed self-organization IoT networking model provides higher efficiency for constructing IoT networks with selforganization scheme in terms of high throughput, low data loss rate and lower control overhead. On the other hand, this scheme has the advantage of the construction of selforganization IoT network.
\end{abstract}

Keywords: Self-Organization Networking, heterogeneous IoT

\section{Introduction}

Recently, heterogeneous IoT environments are developed for supporting IoT service[1]. In heterogeneous IoT environments, any device can be interconnected with and any infrastructure and any devices for the communication. Also, the state of IoT devices change dynamically, e.g., sleeping mode or walk up mode and connected state and/or disconnected state as well as the context of the IoT devices including location and speed. These features look like features of VANET. In particular, VANET is defined with a dynamic network and self-organization IoT network. Here, in order to design self-organization network optimally, the following points should be considered. First at all, coordination function is need to for negotiation between IoT devices. When each IoT device performs self-organization IoT networking in infrastructure-less based IoT environments, IoT device needs network information to construct self-network topology optimally through self-configuration and selfnetworking. Thus, the IoT devices exchange horizontal network information with coordination function. Secondly, each device in IoT networks is defined as constrained nodes which need less configuration. Thus, in order to reduce the signaling about the overhead of configuration for self-organization IoT networking, IoT device can perform less configuration. In this paper, as explained up, considering coordination function and less configuration, novel communication scheme for selforganization IoT networking is proposed. The remainder of the paper is structured as follows. In Section 2, we address related works. And in Section 3, we define the proposed concept and architecture of self-organization IoT networking. Finally, a performance evaluation is provided in Section 4, whereas conclusive remarks are drawn in Section 5.

Received (January 4, 2018), Review Result (March 19, 2018), Accepted (April 3, 2018) 


\section{Related Works}

The self-organization networks (SONs) is defined such as an autonomous networking established by each device, where the device can effectively interact with its peers and perform self-control for network-organization and joining network according to its own status, own service and dynamic network environment in a decentralized infrastructure or infrastructure-less based networks. The similar concept of SONs is proposed by many research areas. For example, SON is defined in 3GPP, IEEE NGSON, ETSI GS AFI, IETF ANIMA WG, IETF AUTOCONF WG. Also, ITU-T defines distributed service networking (DSN) as SONs. Although SONs, autonomic network, ad hoc network, and DSN have been developed in various areas, these technologies have some restrictions to apply in the IoT environments because they don't consider the characteristics of the IoT environments. So, it is needed to study a self-organization IoT networking, including self-organizing network, autonomic network, ad hoc network, and DSN, in general network point of view and for the IoT environments.

In this paper, self-organization IoT networking is further described to enable IoT devices to be configured and managed without the human intervention and fixed/dedicated network facilities. Due to the different characteristics of IoT devic es, the previous studies cannot be directly applied in the IoT environments. To provide self-organization networking in the IoT environments, common characteristics of self-organization networking, architecture of self-organization networking, common requirement, and common functionalities of mechanisms for self-organization networking are described. In order to design self-organization IoT networking optimally in the IoT environment, the following points should be considered.

- Coordination function among the IoT devices: When each IoT device performs self-organization networking in infrastructure-less based IoT environments, the IoT device needs network information to constitute network topology optimally

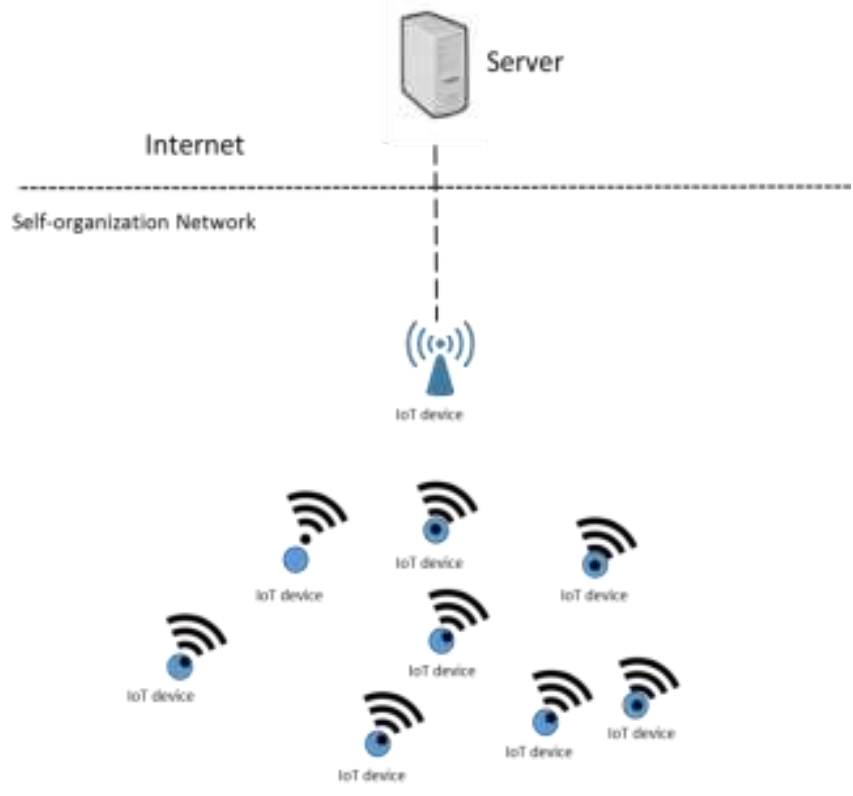

Figure 1. Ad-hoc loT Network (Homogeneous Case) 


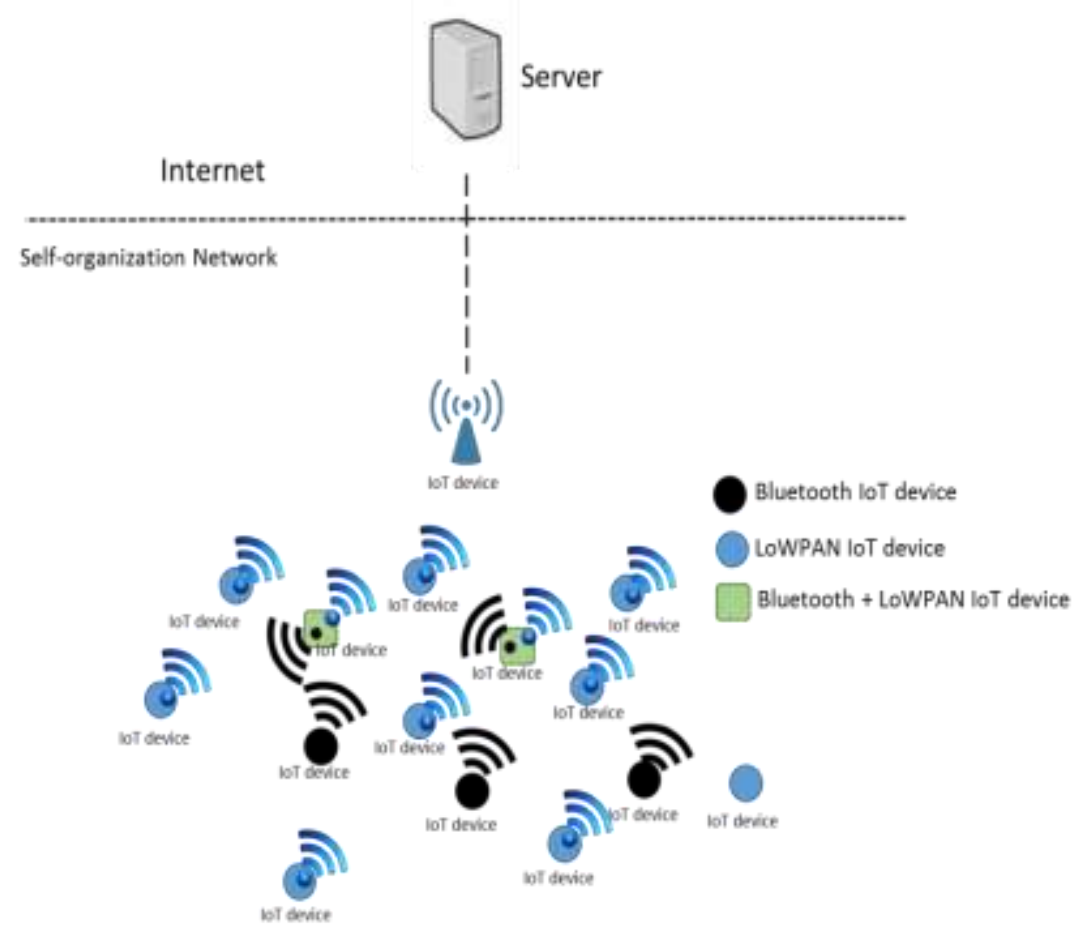

Figure 2. Ad-hoc loT Network (Heterogeneous Case)

through self-configuration. Thus, the IoT devices exchange horizontal network information with coordination function in infrastructure-less based IoT environments.

- Less configuration for self-organization networking: The IoT device could be defined as constrained nodes as well as general network nodes. So it is desired that the overhead of configuration is reduced for self-organization networking. In addition, for less configuration of the IoT device, self-organization networking must be performed with local information such as neighbor device and local IoT service.

- Partially support of IoT services: Generally, service functions are decupled with network functions. In the IoT environments, heterogeneous IoT services are served on heterogeneous IoT devices and these services seems to be dynamic and changed frequently. Due to the requirement of less configuration for selforganization networking in the IoT environments coordination function among the IoT devices and, network functions can do more works for IoT services.

However, in the IoT environment, network configuration and conditions are mainly different to existing ones. In a network point of view, the deployment of, network size, the number of device in network, connectivity between devices, communication type such as multi-hop ad-hoc or single-hop ad-hoc, traffic pattern, security level, mobility support and QoS support are considered for self-organization IoT networking In the IoT environments, the usage of some specific network entities is not possible. Then, not only infrastructure-based IoT networks but also ad-hoc IoT networks can be widely used. In figure 1, an ad-hoc IoT network is depicted in homogeneous case where all IoT device have the network interface of the same wireless technology. An IoT gateway is used to provide the connectivity between a local network and a global network. However, other network entities such as base station/access point are not used to deploy ad-hoc IoT network in the local network. Figure 2 describes an ad-hoc 
IoT network in heterogeneous case in which there are all IoT devices with difference wireless technologies.

\section{Self-organization Networking for the IoT Environments}

\subsection{Concept of Self-Organization Networking}

The purpose of deploying a self-organization IoT network (SOIoTN) in the IoT environments is to provide an autonomous networking by cooperation of each IoT devices with the minimizing of the help of a coordinator in a network for acquiring the service that they want. The overall concept of self-organization IoT networking is shown in figure 3. The functions for self-organization IoT networking are distributed and enabled to each mobile IoT nodes in a network and these functions exchange the necessary information for constructing and maintain a self-network. To do this, all IoT nodes in IoT network are classified into 3 groups by their roles.

- IoT device

- IoT coordination

- IoT gateway

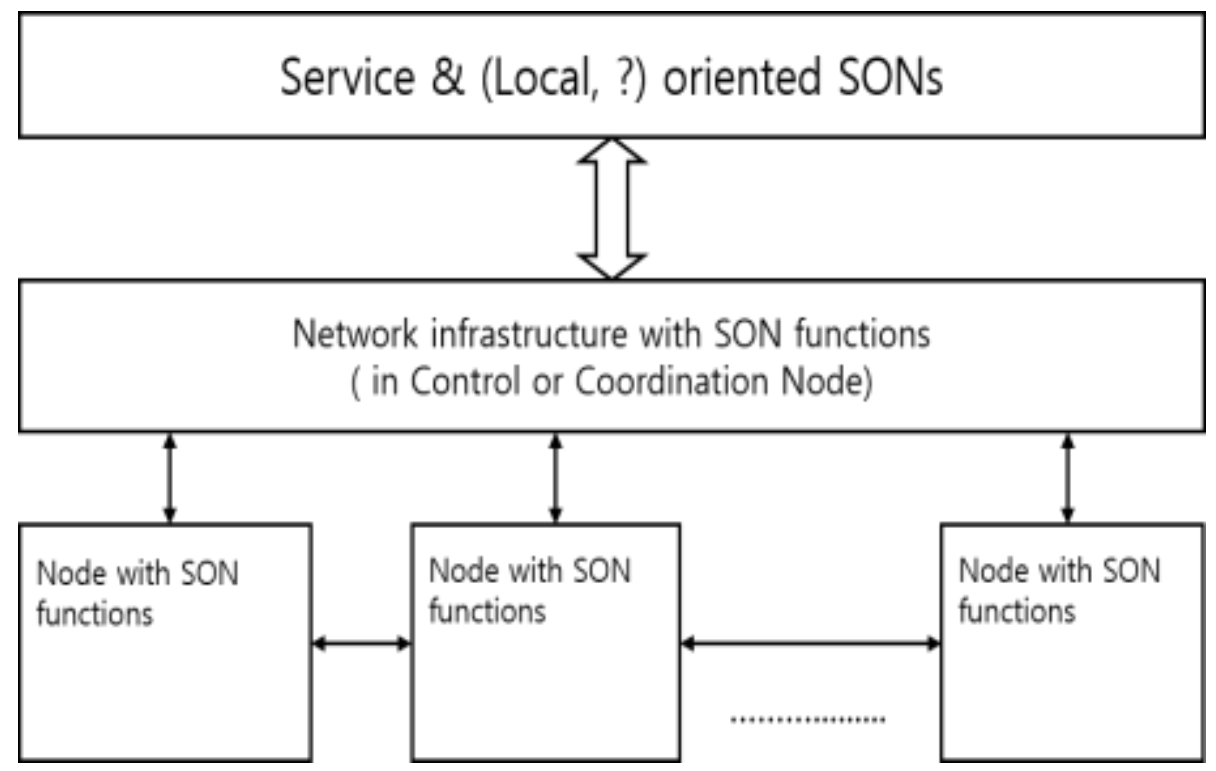

Figure 3. The Concept of Self-Organization loT Networking

First at all, an IoT coordination includes control and coordination functions to construct self-organization IoT network for other IoT devices. During the construction of a selforganization IoT network, the role of each IoT node is determined by their roles and these determinations are defined as a service in the IoT network. An IoT coordination utilizes the information to construct a self-organization IoT network by their roles. These operations are preceded as autonomic and the described functions of self-organization IoT networking will be applied to support these operations. The conventional network entities are classified into a network server node (e.g., router, gateway, server, etc.) and a network client node (such as host, end node, etc.) as their functionalities. And the roles of the conventional network entities are not changed. However, networking entities in the IoT environments are a different. Because of heterogeneous types and capabilities of IoT devices, the conventional network paradigm cannot be used in the IoT environments. 


\subsection{Architecture Model for self-organization IoT Networking}

In the subsection, the architecture model for SOIoTN is proposed as shown in figure 4. First at all, we assume that IoT devices have different network interfaces and different service discovery protocols in local IoT network. Figure 4 shows high-level architecture model for the optimized self-organization IoT networking topology construction which reflects network or service requirements in the IoT environments. The architecture model is comprised of three-parts:

- Network \& service requirement

- Physical network \& service resources

- Self-organization IoT networking(SOIoTN) control/management.

Network \& service requirement part performs a role which is to define the properties reflecting the demand of network operator or service provider. Physical network \& service resources part performs a role which is to define the network interface types and service types in the IoT environments. Self-organization control/management part performs a role that is to construct network topology optimally with network \& service requirements and physical network \& service resource requirements. In this architecture model, the key part is the self-organization control/management. This includes three main functions:

- Self-organization IoT networking(SOIoTN) optimization

- Self-organization IoT networking(SOIoTN) coordination

- Self-organization IoT networking(SOIoTN) recognition

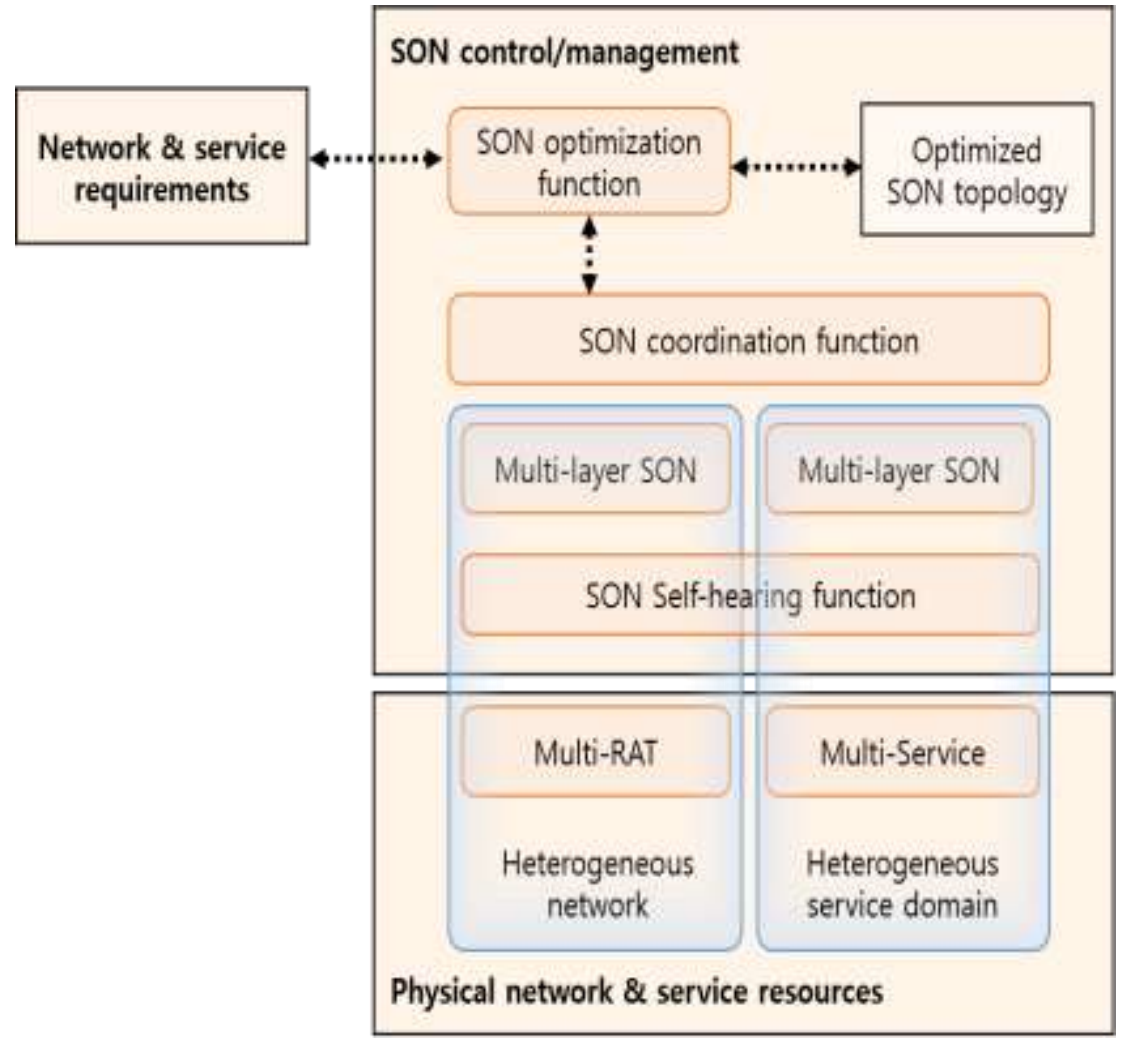

Figure 4. The Architecture Model of Self-Organization Networking 
First at all, the SOIoTN recognition function collects the information about physical network and service resource defined in physical network \& service resource part. The SOIoTN coordination function constructs each multi-layer SOIoTN in heterogeneous IoT networks and heterogeneous service domain with the collected information. Finally, the SOIoTN optimization function constructs each optimized networking topology based on each multi-layer SOIoTN constructed by the SOIoTN coordination function. Or, it constructs the integrated optimal SOIoTN topology based on each multi-layer SOIoTN constructed by the SOIoTN coordination function with considering network \& service requirement of network operator or service provider. Also, the SOIoTN optimization function has the mechanism for optimized SOIoTN topology constitution. The functionality model of SOIoTN in IoT device is proposed as shown in Figure 5. As shown in Figure 5, the functionality in IoT device $(\mathrm{CN})$ consists of the SON information part, the SON coordination part and the SON optimization part. Also, functionality model is highlevel model for the SOIoTN topology construction which reflects network or service requirements in the IoT environments.

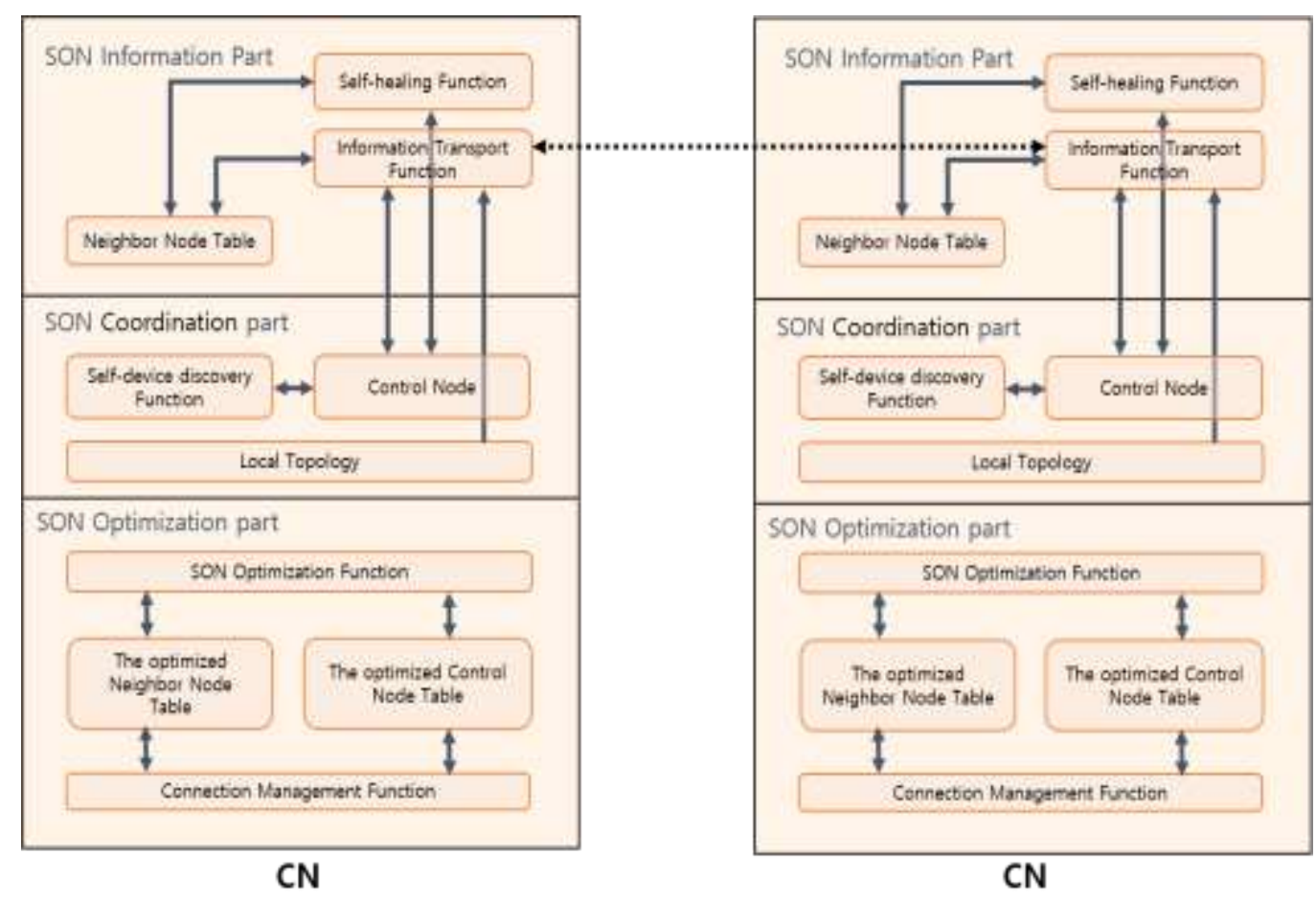

Figure 5. The Functionality Model of SOloTN in loT Device

\section{Performance Evaluation}

To evaluate the performance of the proposed SOIoTN, the performance of the SOIoTN is compared with SON scheme using ad-hoc routing protocol used in general ad-hoc network environment in static random topology. And in the simulations, in order to consist of real IoT environments, we consider two cases; homogeneous case, in which there are all IoT devices with the same wireless technology, and heterogeneous case, in which there are all IoT devices with difference wireless technologies. We use the ns-3 simulator for the simulation. In static random topology environment, the random topology is considered. Where 16 nodes with dual network interfaces are located in $1000 \mathrm{~m} \times 1000 \mathrm{~m}$ square regions. There are 20,30 and 40 flows which have different transmission rate and different starting time. The packet size of all flows is 1000-byte. The transmission rate of each flow is controlled through varying packet interval per packet. The metrics to evaluate the performance are the average data delay and the average throughput. 


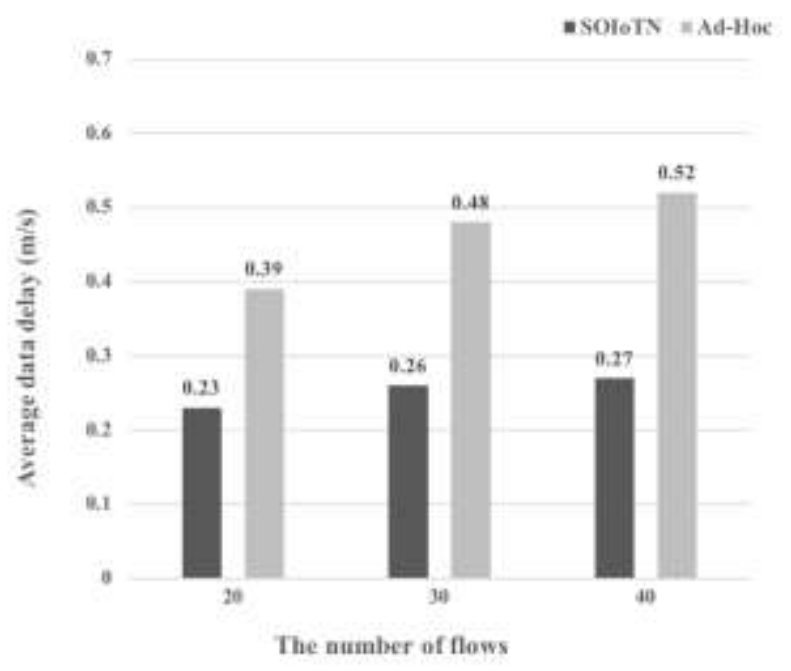

Figure 6. Average Data Delay in Homogeneous Case

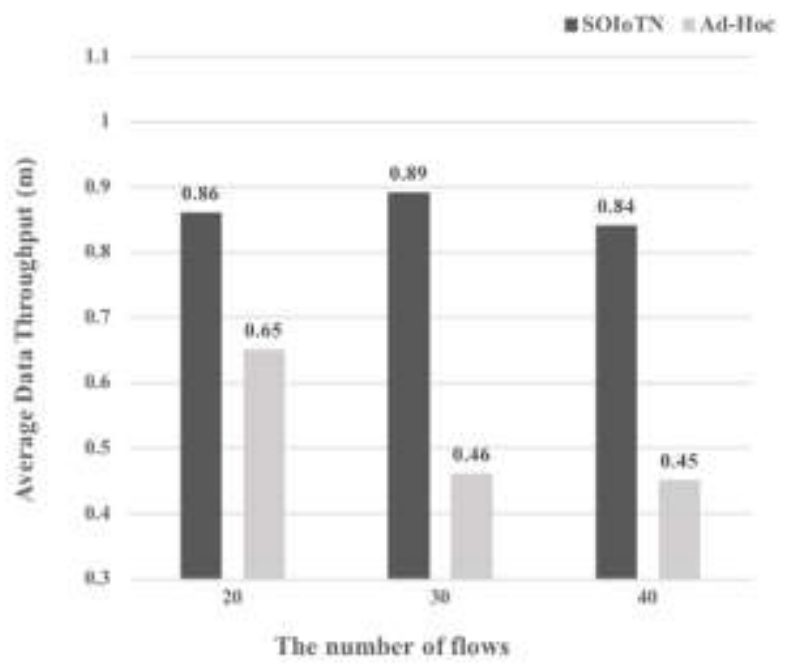

Figure 7. Average Data Throughput in Homogeneous Case

The simulation runs for 200s. The simulation results of homogeneous case are shown in Figure 6 and Figure 7. There shows the performance improvement in the simulation results of the network topology consisting of IoT devices which are enabled to use SOIoTN, compared with the simulation results in general ad-hoc network using only ad-hoc routing protocol such as AODV routing protocol. This shows that SOIoTN can construct better self-IoT network than IoT network using ad-hoc routing protocol through SOIoTN scheme in homogeneous IoT environments. The simulation results of heterogeneous case are shown in figure 8 and figure 9. This shows that SOIoTN can distribute data transmission through dual-network interface in heterogeneous IoT environments. In addition, in this works, the data transmission stability, DTS, is defined to evaluate reliable transmission ratio (RTR) and low transmission delay (LTD) with the total data loss rate during the data transmission and data transmission delay. When the loss rate is less than 5\%, the DTS is good but when loss rate is between $5 \%$ and $10 \%$, the DTS is medium. When loss rate exceeds $15 \%$, the DTS is poor. We obtain good stability (DTS $=4.3 \%$ ), when using 
SOIoTN in heterogeneous IoT environments. However, when IoT device uses only ad-hoc routing protocol such as AODV routing protocol in heterogeneous IoT environments, low

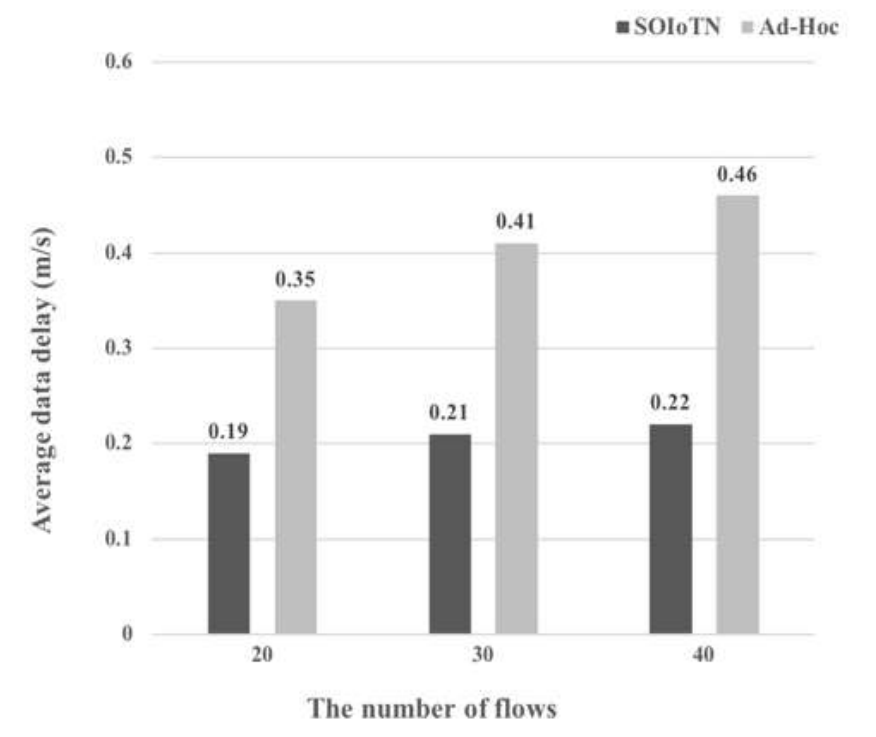

Figure 8. Average Data Delay in Heterogeneous Case

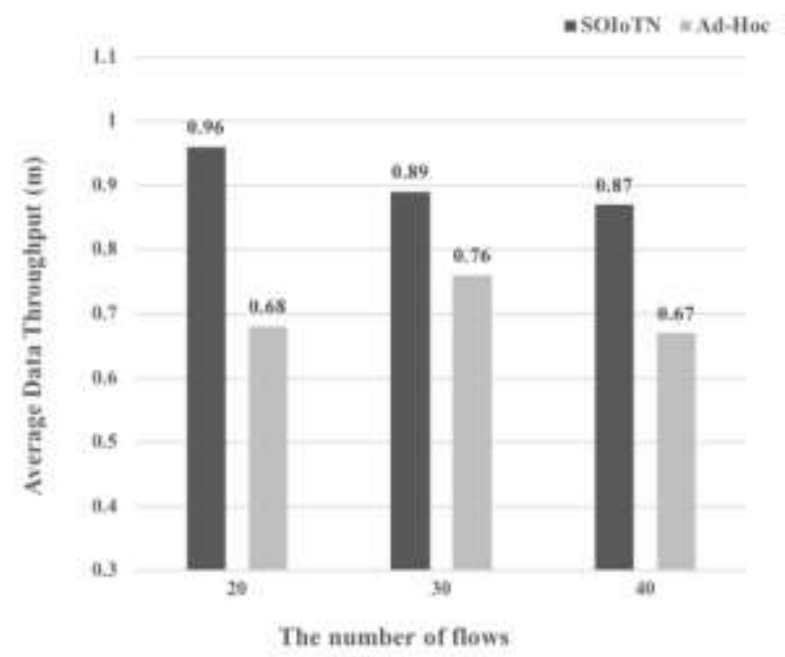

Figure 9. Average the Average Throughput in Heterogeneous Case

stability (DTS $=18 \%$ ) is obtained. By comparison, SOIoTN gives better stability for data transmission than the scheme using only AODV routing protocol. In addition, the results show that SOIoTN gets better service in terms of the stability for data transmission. In order to evaluate more realistic performance of SOIoTN, the simulations run in dynamic IoT environments where 20 mobile nodes with mobility are located randomly and have mobility in $1000 \mathrm{~m} \times 1000 \mathrm{~m}$ square regions. The simulations are evaluated in two same scenarios above. One is evaluated in homogeneous IoT environments. The other is evaluated in the heterogeneous IoT environments using both dual-network interfaces which are IEEE 802.11n network interface and IEEE 802.11p network interface. In the simulations, there are 2-flows with E2E bandwidth requirement of $0.2 \mathrm{Mbps}$, 2-flows with 


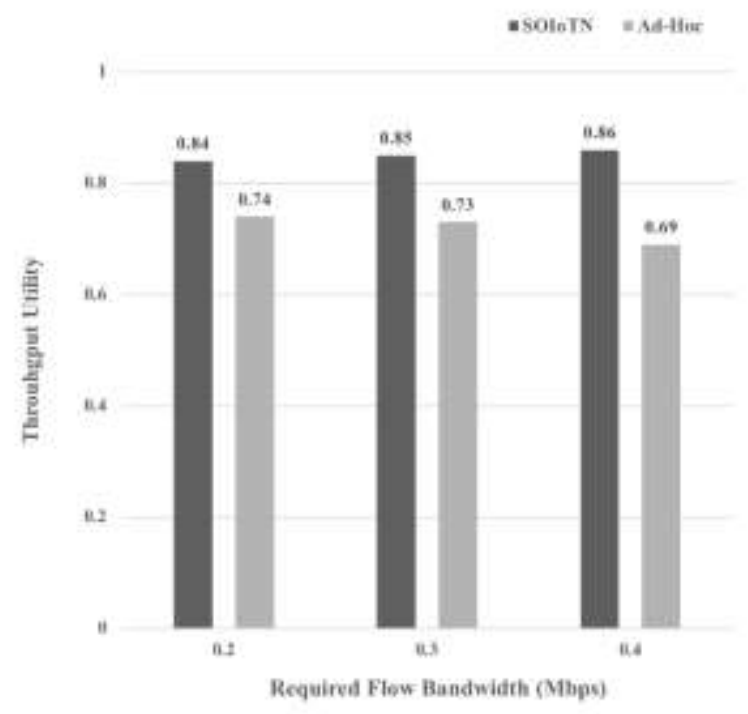

Figure 10. The Throughput Utilities in the Homogeneous loT Environments

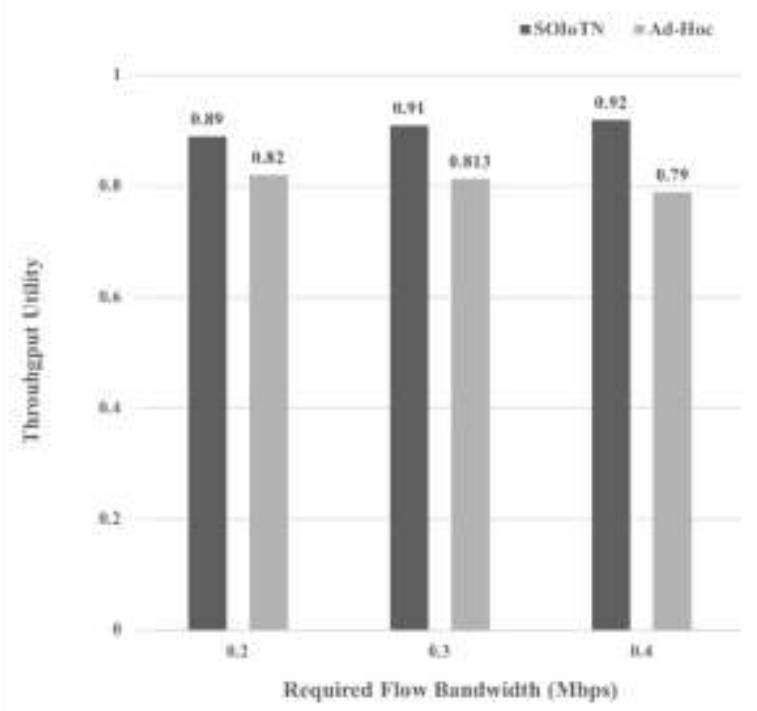

Figure 11. The Throughput Utilities in Heterogeneous loT Environments

E2E bandwidth requirement of $0.3 \mathrm{Mbps}$ and 2-flows with E2E bandwidth requirement of 0.4 Mbps. The E2E pair is chosen randomly. The simulations go for 200s. In the simulations, the metrics used in measuring the throughput utility. The throughput utility is $\min (1$, Tact/Treq). Treq is the upper bound throughput of bandwidth requirement and Tact is the measured throughput. Figure 10 shows the throughput utility in the simulation for the homogeneous IoT environments. As shown in the simulation results, the results in the IoT network adapting SOIoTN show the high throughput utility, while the results in the IoT network adapting ad-hoc routing scheme gets the low throughput utility. In the other words, the SOIoTN scheme also shows better a stable data transmission than scheme using only ad-hoc routing. Also, the same results are shown in the simulation of the heterogeneous IoT environments. As shown in Figure 11, the IoT network adapting SOIoTN shows the high throughput utility, while the IoT network adapting ad-hoc routing scheme gets the low throughput utility. 


\section{Conclusion}

In this paper, the concept and the architecture model for the self-organization IoT networking is proposed to support the self-construction of IoT network at network-level in homogeneous and heterogeneous IoT environments. As explained up, although various kind of self-organization networking schemes exist for IoT environments, they cannot support the dynamic IoT network with mobility such as VANET efficiently and thus be suitable for homogeneous and heterogeneous IoT environments. By comparison, the proposed self-organization IoT networking scheme provides higher efficiency for constructing IoT networks with self-organization scheme in terms of lower overhead. On the other hand, this scheme has the advantage of compatibility for traditional networks.

\section{Acknowledgments}

This research was partly supported by KATS/KEIT (No. 10053677) and by Basic Science Research Program through the National Research Foundation of Korea (NRF) funded by the Ministry of Education (NRF-2017R1D1A1B03034689). This paper is a revised and expanded version of a paper entitled [Communication Scheme for SelfOrganization IoT Networking] presented at [AST 2018, Ho Chi Minh, Vietnam, February $1-3,2018]$.

\section{References}

[1] J. Youn, Y-H. Choi and Y-G. Hong, "The overview of IETF technology standard for IoT", Information and Communications Magazine (Information and Communication), vol. 31, no. 9, (2014) September, pp. 32-39.

[2] J. Youn, "Zone based Ad Hoc Network Construction Scheme for Local IoT Networks", Journal of The Korea Society of Computer and Information, vol. 22, no. 12, (2017), pp. 95-100.

\section{Author}

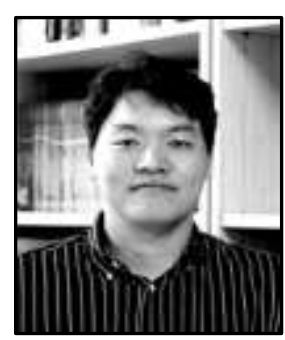

Joosang Youn, is currently associate professor in Dept. of Industrial ICT Engineering at Dong-Eui University since 2008. He received the B.S., M.S. and Ph.D. degrees from Korea University, Seoul, Korea in 2001, 2003 and 2008, respectively. His current research interests include the IoT, 5G, VANET and future network architecture. 